\title{
Probabilities of consistent election outcomes with majorities based on difference in support
}

\begin{abstract}
Computer simulations are used to evaluate the likelihood of consistent outcomes under the class of majorities based on difference in support. These majorities require certain consensus in collective preferences to declare an alternative as the winner. More precisely, individuals show preference intensities in the unit interval among each pair of alternatives and it is required that the winner alternative obtains a difference in the sum of the intensities with respect to the loser alternative. This difference is a real number located between 0 and the total number of voters. We introduce the values of the required threshold for which majorities based on difference in support lead to transitive and triple-acyclic collective decisions with a probability of 1 . Our results improve the previous theoretical ones since they require softer thresholds to reach consistent collective decisions.
\end{abstract}

Keywords: Computer simulations; Majorities based on difference in support; Probability; Transitivity; Triple-acyclicity.

\section{Introduction}

Many consistent conditions such as the transitivity are imposed as minimal requirement concerning any social choice rule used to aggregate individual preferences into a collective outcome. Transitivity represents the idea that, for an individual or collective preference, when alternative $x_{1}$ is preferred to $x_{2}$ and $x_{2}$ is preferred to $x_{3}$ then $x_{1}$ is preferred to $x_{3}$. The literature related to this notion dates back 200 years to the great work of the Marquis de Condorcet (1785) which states that voters with individual transitive rankings can produce an election outcome which is not transitive if one chooses pairwise majority voting as aggregation. This result gave rise to numerous studies and promoted the probabilistic study of the occurrence of voting paradoxes and their consequences under different aggregation rules. 
The probability of voting paradoxes has been the subject of a whole strand of literature. Under this approach, it is assumed an a priori probability model to describe the individual preferences, derived the conditions under which the paradox appears and reached probabilities through combinatoric calculus. In this context, stand out the studies about majority rule (Fishburn and Gehrlein 1980; Gehrlein 1983; Gehrlein and Fishburn 1976), supermajority rules (Balasko and Crès 1997; Tovey 1997) or scoring rules (Cervone et al 2005; Gehrlein and Fishburn 1980, 1981, 1983), among others. To circumvent the impossibility of having analytical results, other papers have undertaken a methodology using computer simulations of elections. Specifically, the study of the cyclical and intransitive collective decisions under the simple majority rule are carried out in Campbell and Tullock (1965); DeMeyer and Plott (1970); Jones et al (1995); Klahr (1966), among others.

Voting rules based on the majority principle are the most studied in the literature and that principle remains one of the most widely used voting rules in real life. The majority rule, and other classic voting systems, have the advantage of being simple to use and easy to understand by the voters but they have major drawbacks. One of them is based on the idea that the preferences expressed by the voters are assumed to be dichotomous (also called crisp or ordinal), i.e. individuals can only declare if an alternative is preferred to another, or if they are indifferent. As the Nobel laureate Amartya Sen (1970) has noted, "voters opinions can be misrepresented when the preferences are dichotomous in the sense that the collective decision does not take into consideration the intensities of each individual preference". The importance of considering intensities in the individual preferences has also been noted in Cook and Kress (1985); Meek (1975); Nurmi (1981, 2008); Tanino (1984), and Morales (1797) (see translation in McLean and Urken (1995)), among others.

Reciprocal preferences have been introduced in the literature in order to deal with preference intensities. This framework allows individuals to show preference intensities among each pair of alternatives by a real number in the unit interval $[0,1]$. It is clear that the information contained in reciprocal preferences is much richer than the case of dichotomous preferences. Indeed, for each voter, a reciprocal preference between two alternatives $x_{1}$ and $x_{2}$ expresses the degree by which the voter prefers $x_{1}$ to $x_{2}$. More precisely, the closer the intensity is to 1 , the more $x_{1}$ is preferred to $x_{2}$, and the closer the intensity is to 0 , the more $x_{2}$ is preferred to $x_{1}$. In addition, an intensity of 0.5 stands for the exact indifference between the two alternatives $x_{1}$ and $x_{2}$. Following this approach, when we extend the set of alternatives from two to three alternatives, we assume that each individual reciprocal preference fulfil some properties of transitivity in order to guarantee individual rationality of each reciprocal preference.

Considering preference intensities, majorities based on difference in support $\widetilde{M}_{k}$ have been introduced in the literature as a possible aggregation system. These majorities require certain consensus in collective preferences to declare an alternative as the winner and depend on the idea of a threshold. More precisely, these aggregation rules suggest that alternative $x_{1}$ is preferred 
to alternative $x_{2}$ if and only if the sum of the intensities for $x_{1}$ exceeds the aggregated intensity of $x_{2}$ by a threshold $k$ given by a real number located between 0 and the total number of voters. In other words, a higher value of $k$ means that a stronger preference for $x_{1}$ over $x_{2}$ is needed in order to $x_{1}$ to be declared socially preferred to $x_{2}$. Conversely, a low value of $k$ means that a weak preference for $x_{1}$ over $x_{2}$ is needed to declare $x_{1}$ socially preferred to $x_{2}$. The axiomatic characterization of majorities based on difference in support is introduced in García-Lapresta and Llamazares (2010). Notice that, for other kinds of operators which can be used for the aggregation of reciprocal preferences and their characterizations, the reader is referred to García-Lapresta and Llamazares (2000); Llamazares (2004, 2007); Llamazares and García-Lapresta (2003, 2008), among others.

This paper is devoted to analyze the probabilities of consistent collective decisions over the class of majorities based on difference in support. We specifically calculate the probabilities of transitive and triple-acyclic strict collective preferences and the corresponding ones of transitive weak collective preferences for these majorities. To calculate these probabilities, we apply the Monte-Carlo simulation methodology inspired by the studies in Campbell and Tullock (1965); DeMeyer and Plott (1970); Jones et al (1995); Klahr (1966), among others.

Specifically, we generate the individual reciprocal preference relations for the case of three alternatives. Each individual intensity of preference is understood as a continuous random variable in the unit interval consistently built with a specific transitivity condition over the individual's reciprocal preference relations. Then, we fix the required difference in support and aggregate these individual preferences with the corresponding majority based on difference in support. We derive the resultant collective ordering of alternatives and evaluate its consistency. Finally, we iterate that procedure to estimate desired probabilities as the number of consistent orderings over the total number of simulated collective orderings.

The objective of this paper is twofold. First, the methodology proposed here allows us to hypothesize about a relationship between the type of individual preference intensities and the likelihood of consistent collective decisions. Intuitively, we expected to find softer conditions to have consistent collective decisions with probability 1 as individuals are more rational, i.e. the strongest the assumed individual transitivity condition is. As we will see, our results refute that idea. Second, we compare our results on probabilities with the theoretical ones about the consistency of the collective preferences under majorities based on difference in support in Llamazares et al (2013) and Llamazares and Pérez-Asurmendi (2015). In these articles, necessary and sufficient conditions for which these majorities provide transitive and tripleacyclic collective decisions have been introduced. The algebraic tools used in the proofs lead to several impossibility results. Specifically, for the case of the transitivity of the collective decisions they proved that it can be always found an example of intransitive collective preferences when the individual transitivity condition is weak. In the case of assuming stronger conditions to individual 
preferences, the collective preference is transitive only when individual preferences show a higher level of unanimity. Although they found less demanding conditions in their results about the triple-acyclicity of the collective decision, it is also required a high level of similarity in individual preferences in order to find consistent collective decisions. Under the probabilistic approach followed here, the required conditions for having consistent collective decisions are much softer than there promoting the use of these types of majorities whenever the consistency of the collective decision is a concern for the society or the policy maker.

The paper is organized as follows. Section 2 describes the theoretical framework followed in this paper and introduces majorities based on difference in support. Section 3 is devoted to the method of simulation. Sections 4 and 5 discuss the results and Section 6 concludes.

\section{Preliminaries}

Consider a set of alternatives $X=\left\{x_{1}, x_{2}, x_{3}\right\}$ in an election with $m$ individuals. Let $S$ be a binary relation on $X$, i.e. a subset of the Cartesian product $X \times X$. In what follows, $x_{i} S x_{j}$ stands for $\left(x_{i}, x_{j}\right) \in S$, i.e. when $x_{i}$ is in the relation $S$ with $x_{j} . S^{-1}$ is the inverse relation of $S$ defined by $x_{i} S^{-1} x_{j} \Leftrightarrow x_{j} S x_{j}$ and $S^{c}$ is the complement relation of $S$ defined by $x_{i} S^{c} x_{j} \Leftrightarrow \neg\left(x_{i} S x_{j}\right)$. Given two binary relations $S$ and $T$, the intersection of $S$ and $T$ is also a binary relation defined by $x_{i}(S \cap T) x_{j} \Leftrightarrow\left(x_{i} S x_{j} \wedge x_{i} T x_{j}\right)$. A binary relation $S$ on $X$ is

1. reflexive if $\forall x \in X, x S x$,

2. symmetric if $\forall x_{i}, x_{j} \in X, x_{i} S x_{j} \Rightarrow x_{j} S x_{i}$,

3. asymmetric if $\forall x_{i}, x_{j} \in X, x_{i} S x_{j} \Rightarrow \neg\left(x_{j} S x_{i}\right)$,

4. antisymmetric if $\forall x_{i}, x_{j} \in X,\left(x_{i} S x_{j} \wedge x_{j} S x_{i}\right) \Rightarrow x_{i}=x_{j}$,

5. complete if $\forall x_{i}, x_{j} \in X, x_{i} S x_{j} \vee x_{j} S x_{i}$,

6. transitive if $\forall x_{i}, x_{j}, x_{l} \in X,\left(x_{i} S x_{j} \wedge x_{j} S x_{l}\right) \Rightarrow x_{i} S x_{l}$,

7. triple-acyclic if $\forall x_{i}, x_{j}, x_{l} \in X,\left(x_{i} S x_{j} \wedge x_{j} S x_{l}\right) \Rightarrow \neg\left(x_{l} S x_{i}\right)$.

A weak preference $R$ is a complete binary relation on the set of alternatives $X$. The strict preference $P$ associated with $R$ is the asymmetric binary relation on $X$ defined by $P=\left(R^{-1}\right)^{c}$ and the corresponding indifference relation $I$ is the reflexive and symmetric binary relation on $X$ defined by $I=R \cap R^{-1}$. $\mathcal{P}(X)$ is the set of strict preferences. A weak ordering is a transitive weak preference whereas a linear ordering is also antisymmetric.

From definitions above it is well known that any weak ordering implies a transitive strict preference relation and a transitive indifference relation. Moreover, any transitive strict preference is also a triple-acyclic preference relation. Notice that the converse is not true.

Given that the social decision between two alternatives is given by either a strict preference relation or an indifference relation, and that three alternatives are in contest, we consider the 27 cases in Table 1 as possible social outcomes. 
Table 1 Possible social outcomes in a three-alternative election.

\begin{tabular}{lllllllll}
\hline 1. $x_{1} P x_{2}$ & $x_{2} P x_{3}$ & $x_{1} P x_{3}$ & 10. $x_{1} I x_{2}$ & $x_{2} P x_{3}$ & $x_{1} P x_{3}$ & 19. $x_{1} I x_{2}$ & $x_{2} P x_{3} x_{1} I x_{3}$ \\
2. $x_{1} P x_{3}$ & $x_{3} P x_{2}$ & $x_{1} P x_{2}$ & 11. $x_{2} I x_{3}$ & $x_{3} P x_{1}$ & $x_{2} P x_{1}$ & 20. $x_{1} P x_{2}$ & $x_{2} P x_{3}$ & $x_{1} I x_{3}$ \\
3. $x_{2} P x_{1}$ & $x_{1} P x_{3}$ & $x_{2} P x_{3}$ & 12. $x_{1} I x_{3}$ & $x_{3} P x_{2}$ & $x_{1} P x_{2}$ & 21. $x_{3} P x_{1}$ & $x_{1} P x_{2}$ & $x_{2} I x_{3}$ \\
4. $x_{2} P x_{3}$ & $x_{3} P x_{1}$ & $x_{2} P x_{1}$ & 13. $x_{1} I x_{2}$ & $x_{2} I x_{3}$ & $x_{1} I x_{3}$ & 22. $x_{2} P x_{3}$ & $x_{3} P x_{1}$ & $x_{1} I x_{2}$ \\
5. $x_{3} P x_{1}$ & $x_{1} P x_{2}$ & $x_{3} P x_{2}$ & 14. $x_{1} P x_{2}$ & $x_{2} I x_{3}$ & $x_{1} I x_{3}$ & 23. $x_{3} P x_{2}$ & $x_{1} P x_{3}$ & $x_{1} I x_{2}$ \\
6. $x_{3} P x_{2}$ & $x_{2} P x_{1}$ & $x_{3} P x_{1}$ & 15. $x_{1} I x_{2}$ & $x_{2} I x_{3}$ & $x_{1} P x_{3}$ & 24. $x_{2} P x_{1}$ & $x_{1} P x_{3}$ & $x_{2} I x_{3}$ \\
7. $x_{1} P x_{2}$ & $x_{2} I x_{3}$ & $x_{1} P x_{3}$ & 16. $x_{1} I x_{2}$ & $x_{2} I x_{3}$ & $x_{3} P x_{1}$ & 25. $x_{3} P x_{2}$ & $x_{2} P x_{1}$ & $x_{1} I x_{3}$ \\
8. $x_{2} P x_{1}$ & $x_{1} I x_{3}$ & $x_{2} P x_{3}$ & 17. $x_{1} I x_{3}$ & $x_{3} P x_{2}$ & $x_{1} I x_{2}$ & 26. $x_{1} P x_{2}$ & $x_{2} P x_{3}$ & $x_{3} P x_{1}$ \\
9. $x_{3} P x_{1}$ & $x_{1} I x_{2}$ & $x_{3} P x_{2}$ & 18. $x_{2} P x_{1}$ & $x_{1} I x_{3}$ & $x_{2} I x_{3}$ & 27. $x_{2} P x_{1}$ & $x_{1} P x_{3}$ & $x_{3} P x_{2}$ \\
\hline
\end{tabular}

Our interest focuses on the frequency of consistent social outcomes given the 27 possible outcomes above. We distinguish among three cases of consistent outcomes; the case of weak orderings corresponding to the first thirteen outcomes, the case of transitive strict preferences corresponding to the first nineteen and the case of triple-acyclic strict preferences corresponding to the first twenty-fifth outcomes.

\subsection{Individual preferences}

We consider that individuals compare the alternatives on $X$ by pairs and declare their preferences by means of values $r_{i j}^{p}$ which belong to the unit interval $[0,1]$ with the following interpretation: $r_{i j}^{p}>0.5$ indicates that the individual $p$ prefers the alternative $x_{i}$ to the alternative $x_{j}$, the more the nearer is the value of $r_{i j}^{p}$ to 1 that represents the maximum degree of preference for $x_{i}$ over $x_{j}$; conversely, $r_{i j}^{p}<0.5$, means that individual $p$ prefers alternative $x_{j}$ to $x_{i}$, the more the nearer is the value of $r_{i j}^{p}$ to 0 that represents the maximum degree of preference for $x_{j}$ over $x_{i}$; finally, $r_{i j}^{p}=0.5$ stands for the indifference between $x_{i}$ and $x_{j}$ for individual $p$. The reciprocity of these preferences is described by the condition $r_{i j}^{p}+r_{j i}^{p}=1$.

As noted in the Introduction, to avoid the possibility of having incoherent individual preferences, we need to assume some kind of rationality condition. But, in this framework, several concepts could be taken to ensure such rationality requirement (see, among others, Dasgupta and Deb 1996; Dubois and Prade 1980; García-Lapresta and Meneses 2005; Zadeh 1971). Here, we consider the following transitivity conditions for reciprocal preference relations.

Definition 1 We say that individual $p$ is

1. 0.5 -transitive if $\forall i, j, l \in\{1,2,3\}$

$$
\left(r_{i j}^{p}>0.5 \wedge r_{j l}^{p}>0.5\right) \Rightarrow r_{i l}^{p}>0.5,
$$

2. min-transitive if $\forall i, j, l \in\{1,2,3\}$

$$
\left(r_{i j}^{p}>0.5 \wedge r_{j l}^{p}>0.5\right) \Rightarrow r_{i l}^{p} \geq \min \left\{r_{i j}^{p}, r_{j l}^{p}\right\},
$$


3. am-transitive if $\forall i, j, l \in\{1,2,3\}$

$$
\left(r_{i j}^{p}>0.5 \wedge r_{j l}^{p}>0.5\right) \Rightarrow r_{i l}^{p} \geq\left(r_{i j}^{p}+r_{j l}^{p}\right) / 2
$$

4. $\max$-transitive if $\forall i, j, l \in\{1,2,3\}$

$$
\left(r_{i j}^{p}>0.5 \wedge r_{j l}^{p}>0.5\right) \Rightarrow r_{i l}^{p} \geq \max \left\{r_{i j}^{p}, r_{j l}^{p}\right\}
$$

The preferences of each individual over the alternatives in $X=\left\{x_{1}, x_{2}, x_{3}\right\}$ can be represented using a $3 \times 3$ matrix $R^{p}=\left(r_{i j}^{p}\right)$ as follows:

$$
R^{p}=\left(\begin{array}{ccc}
0.5 & r_{12}^{p} & r_{13}^{p} \\
1-r_{12}^{p} & 0.5 & r_{23}^{p} \\
1-r_{13}^{p} & 1-r_{23}^{p} & 0.5
\end{array}\right)
$$

Individual preferences are collected in a vector where each vector-element represents the preferences of an individual. Assuming $m$ individuals ${ }^{1}$ a profile of reciprocal preferences is a vector $\left(R^{1}, \ldots, R^{m}\right) \in \mathcal{R}(X)^{m}$, where $\mathcal{R}(X)$ the set of all reciprocal preference relations.

\subsection{Majorities based on difference in support}

In García-Lapresta and Llamazares (2010), majorities based on difference in support were introduced in the framework of reciprocal preferences allowing individuals to declare their degrees of preferences over pairs of alternatives. Majorities based on difference in support allow us to aggregate each profile of reciprocal preferences into a strict collective preference $P_{k}$ over the set of alternatives. Under these rules, the winner alternative is required to reach a support that exceeds the support for the other alternative in a quantity, fixed before the voting process. Formal definition for these majorities is as follows.

Definition 2 (Majorities based on difference in support or $\widetilde{M}_{k}$ majorities García-Lapresta and Llamazares (2010)) Given $k \in[0, m)$, the majority based on difference in support or $\widetilde{M}_{k}$ majority is the mapping $\widetilde{M}_{k}: \mathcal{R}(X)^{m} \longrightarrow \mathcal{P}(X)$ defined by $\widetilde{M}_{k}\left(R^{1}, \ldots, R^{m}\right)=P_{k}$, where

$$
x_{i} P_{k} x_{j} \Leftrightarrow \sum_{p=1}^{m} r_{i j}^{p}>\sum_{p=1}^{m} r_{j i}^{p}+k .
$$

Using the fact that $r_{i j}^{p}=1-r_{j i}^{p}$ for each voter $p,(2)$ is equivalent to:

$$
x_{i} P_{k} x_{j} \Leftrightarrow \frac{\sum_{p=1}^{m} r_{i j}^{p}}{m}>0.5+\frac{k}{2 m} \text {. }
$$

1 To calculate the probabilities presented here, $m$ takes the following values: $3,4,5,10$, $100,1,000$ and 100,000. 
The indifference relation associated with $P_{k}$ is defined by:

$$
x_{i} I_{k} x_{j} \Leftrightarrow\left|\sum_{p=1}^{m} r_{i j}^{p}-\sum_{p=1}^{m} r_{j i}^{p}\right| \leq k .
$$

Putting the relation $r_{i j}^{p}=1-r_{j i}^{p}$ into (4), one obtains:

$$
x_{i} I_{k} x_{j} \Leftrightarrow\left|\frac{\sum_{p=1}^{m} r_{i j}^{p}}{m}-0.5\right| \leq \frac{k}{2 m} .
$$

Example 1 Let $R^{I}$ and $R^{I I}$ be the following reciprocal preference relations over the alternatives on $X=\left\{x_{1}, x_{2}, x_{3}\right\}$.

$$
R^{I}=\left(\begin{array}{ccc}
0.5 & 1 & 0.9 \\
0 & 0.5 & 0.6 \\
0.1 & 0.4 & 0.5
\end{array}\right), \quad R^{I I}=\left(\begin{array}{ccc}
0.5 & 0.8 & 1 \\
0.2 & 0.5 & 0.7 \\
0 & 0.3 & 0.5
\end{array}\right)
$$

Consider the profile $\left(R^{1}, R^{2}, R^{3}, R^{4}, R^{5}\right)$ where

$$
R^{p}= \begin{cases}R^{I} & \text { if } p=1,2,3 \\ R^{I I} & \text { if } p=4,5\end{cases}
$$

Assuming a required difference in support $k$ equal to 1.75 and applying the corresponding $\widetilde{M}_{1.75}$ majority we have

$$
\begin{gathered}
\sum_{p=1}^{5} r_{12}^{p}=4.6>\sum_{p=1}^{5} r_{21}^{p}+1.75=0.4+1.75 \Rightarrow x_{1} P_{1.75} x_{2}, \\
\left|\sum_{p=1}^{5} r_{23}^{p}-\sum_{p=1}^{5} r_{32}^{p}\right|=|3.2-1.8| \leq 1.75 \Rightarrow x_{2} I_{1.75} x_{3}, \\
\sum_{p=1}^{5} r_{13}^{p}=4.7>\sum_{p=1}^{5} r_{31}^{p}+1.75=0.3+1.75 \Rightarrow x_{1} P_{1.75} x_{3} .
\end{gathered}
$$

As noticed in the Introduction, in the case of crisp preferences, given a pair of alternatives, individuals declare if they prefer an alternative to another one or if they are indifferent between them. In other words, the values of $r_{i j}^{p}$ are restricted to the set of discrete values $\{0,0.5,1\}$. If $r_{i j}^{p}=1$, individual $p$ prefers alternative $x_{i}$ to alternative $x_{j}$, whereas if $r_{i j}^{p}=0$, individual $p$ prefers $x_{j}$ to $x_{i}$. If $r_{i j}^{p}=0.5$, individual $p$ is indifferent between both alternatives. In this framework, the concept of majorities based on difference of votes was introduced in García-Lapresta and Llamazares (2001) and was later axiomatically characterized in Llamazares (2006), and subsequently in Houy (2007). Under these majorities, an alternative, say $x_{i}$, is declared the winner if the number of individuals who prefer that alternative, to the other one, say $x_{j}$, 
exceeds the number of individuals who prefer $x_{j}$ to $x_{i}$ in a difference of votes, fixed before the election process. With $m$ individuals, that difference could take any integer value in $\{0, \ldots, m-1\}$. It is clear that these majorities are located between simple majority rule when the difference of votes is zero and unanimity when the difference of votes is the total number of individuals $m$ minus one.

Assuming weak or linear individual orderings and using the well-known Impartial Anonymous Culture (IAC) condition (Gehrlein and Fishburn 1976), calculations of the probabilities of consistent outcomes (transitivity and tripleacyclicity) under majorities based on difference in votes have been conducted in Diss and Pérez-Asurmendi (2015). The IAC condition assumes that all voting situations are drawn independently and uniformly given that each voting situation indicates a specific combination of the number of voters associated with each order of the alternatives. The objective of Diss and Pérez-Asurmendi (2015) was twofold. First, the needed thresholds which guarantee that the probability of consistent outcomes is close to 1 have been found. Second, the authors have set forth the impact of weak orderings and linear orderings for the individual preferences on the probability of consistent outcomes. It is shown for instance that in the cases of transitive and triple-acyclic strict preferences, the probabilities are higher considering weak than linear orderings.

In the present paper, we extend the study in Diss and Pérez-Asurmendi (2015) to the majorities based on difference in support $\widetilde{M}_{k}$. However, as long as the intensities of preference between each pair of alternatives can take any value in the continuous interval $[0,1]$, the IAC model can not be applied. Our difficulty is that there is not any equivalent model to IAC in the framework of reciprocal preferences since the set of voting situations is not numerable. In other words, the probabilistic analysis carried out in Diss and Pérez-Asurmendi (2015) turns impossible to study in the case of majorities based on difference in support $\widetilde{M}_{k}$. Consequently, we perform a computer simulation to estimate these probabilities. Our simulation method will follow the same spirit as the Impartial Culture (IC) condition (Guilbaud 1952), a well-known model which considers the set of all preference profiles as a sample space and where a voter preference profile identifies the specific preference ranking that each voter has for the candidates. Notice that individual voter's preferences are not anonymous under IC condition while they are under IAC assumption.

\section{Simulation method}

In this section, we detail the simulation method used in this paper in order to provide the probabilities of reaching consistent collective decisions under $\widetilde{M}_{k}$ majorities. Our probabilities are estimated as the proportion of the number of consistent outcomes in the simulation over the total number of simulated 
outcomes. We generate 100,000 outcomes to guarantee our results with a confidence level of $99 \%$ and a sampling error of less than a $0.0041 \%^{2}$.

In the following, we describe the methodology applied in the simulations to estimate the probability for the considered three types of consistent collective decisions under $\widetilde{M}_{k}$ majorities, i.e. transitive weak preferences, transitive and triple-acyclic strict preferences. We follow that scheme taking into account each type of individual transitive reciprocal relations, i.e. 0.5 -transitive, mintransitive, am-transitive and max-transitive reciprocal preference relations. Notice that the matrix in (1) representing a reciprocal preference relation is determined by the vector composed of the intensities $r_{12}, r_{23}$ and $r_{13}$.

1. At the beginning of the evaluation, we randomly generate $m$ vectors representing the reciprocal preferences of the $m$ individuals. For each individual, each component of the triplet $\left(r_{12}, r_{23}, r_{13}\right)$ is drawn from an uniform distribution on $[0 ; 1]$. Such vectors are built bearing in mind one of the considered transitivity conditions for reciprocal preference relations. In other words, if the resulting individual reciprocal preference $\left(r_{12}, r_{23}, r_{13}\right)$ is not transitive for the function under consideration (i.e. 0.5-transitivity, min-transitivity, am-transitivity and max-transitivity), it is rejected. This first step ends when exactly $m$ individual transitive reciprocal preferences are generated.

2. We compute the sum of the individuals' intensities of preference over each pair of alternatives relative to the number of voters $m$ through a vector $\boldsymbol{S}=\left(\frac{S_{12}}{m}, \frac{S_{23}}{m}, \frac{S_{13}}{m}\right)$ where $S_{i j}=\sum_{p=1}^{m} r_{i j}^{p}$.

3. Having in mind the conditions in equations (3) and (5) and the value of the threshold $a=\frac{k}{2 m}$, the collective decision is evaluated over each pair of alternatives in the vector $\boldsymbol{S}$.

4. The collective decision in $\boldsymbol{S}$ is classified following the cases of possible collective outcomes displayed in Table 1 . For instance, the case 27 corresponds to $\frac{S_{12}}{m}<0.5-a, \frac{S_{23}}{m}<0.5-a$, and $\frac{S_{13}}{m}>0.5+a$. Indeed, if the collective decision is one of the cases 26 or 27 , the strict preference $P_{k}$ is not triple-acyclic. If it is one of the cases from 19 to 27, the strict preference $P_{k}$ is not transitive. Finally, if it is one of the cases from 14 to 27 , the weak preference $R_{k}$ is not transitive.

2 Assuming a proportion of consistent outcomes $P$ on the population of a 50\%, the proportion $p$ in a random sample of size $n \geq 30$ for a confidence level of $99 \%$, diverges from the one of the population in an error of less than $\epsilon$ :

$$
\operatorname{Prob}(|P-p| \leq \epsilon) \geq 0.99
$$

Taken into account that the sample proportion $p$ is distributed as $N(P, \sqrt{P(1-P) / n})$, the sampling error $\epsilon$ is as follows:

$$
\epsilon=z_{\alpha / 2} \sqrt{P(1-P) / n} \text {. }
$$

In our case, $n=100,000$ and the corresponding percentile of the normal distribution for a confidence level of $99 \%$ is $z_{\alpha / 2}=2.57$. Thus, $\epsilon \leq 0.00407$. 
5. This four steps are iterated 100,000 times to obtain the number of inconsistent collective decisions. Specifically, the number of simulated outcomes in which the weak preference $R_{k}$ is transitive, in which the strict preference $P_{k}$ is transitive and in which $P_{k}$ is triple-acyclic, respectively.

6. Finally, each of the desired probabilities, i.e. the probability of transitive $R_{k}$ and the probability of transitive and triple-acyclic $P_{k}$, is calculated as the number of consistent outcomes over the total number of simulated outcomes.

Below, the code used for the evaluation of our probabilities is presented for the case of am-transitive individual reciprocal preferences, a number of voters $m=3$ and a required threshold $k=0.1$.

Simulation code: the case of am-transitive individual reciprocal preferences, $m=3$, and $k=0.1$.

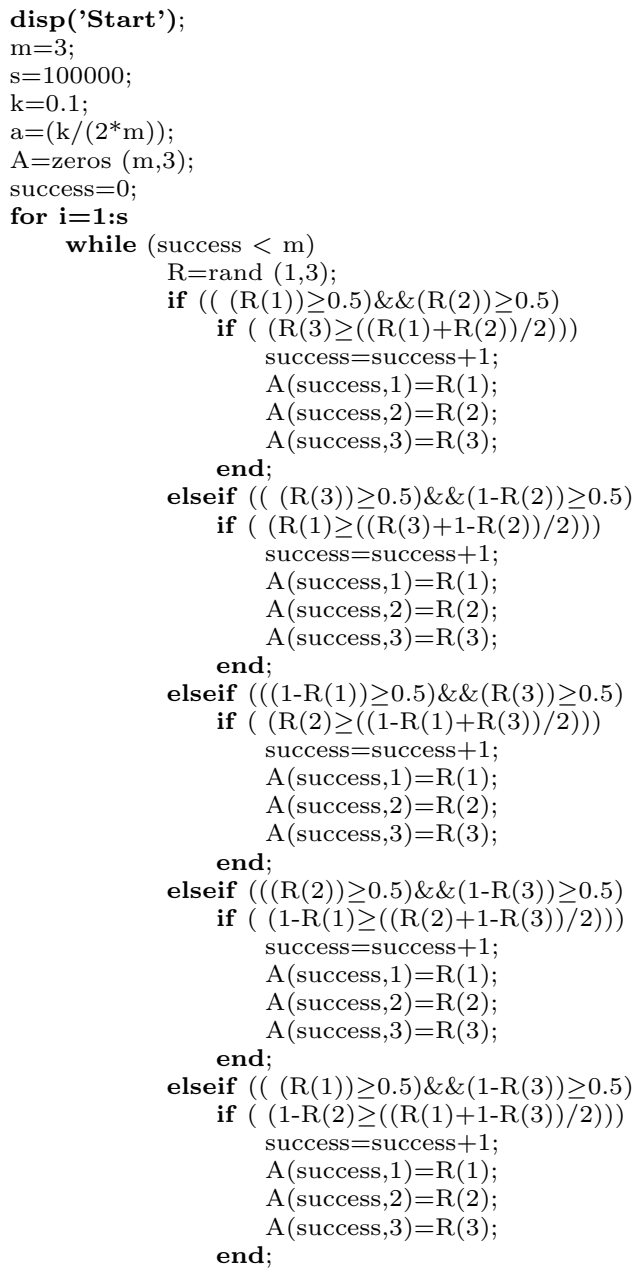




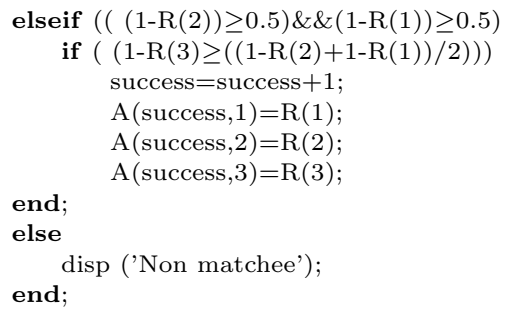

\section{end;}

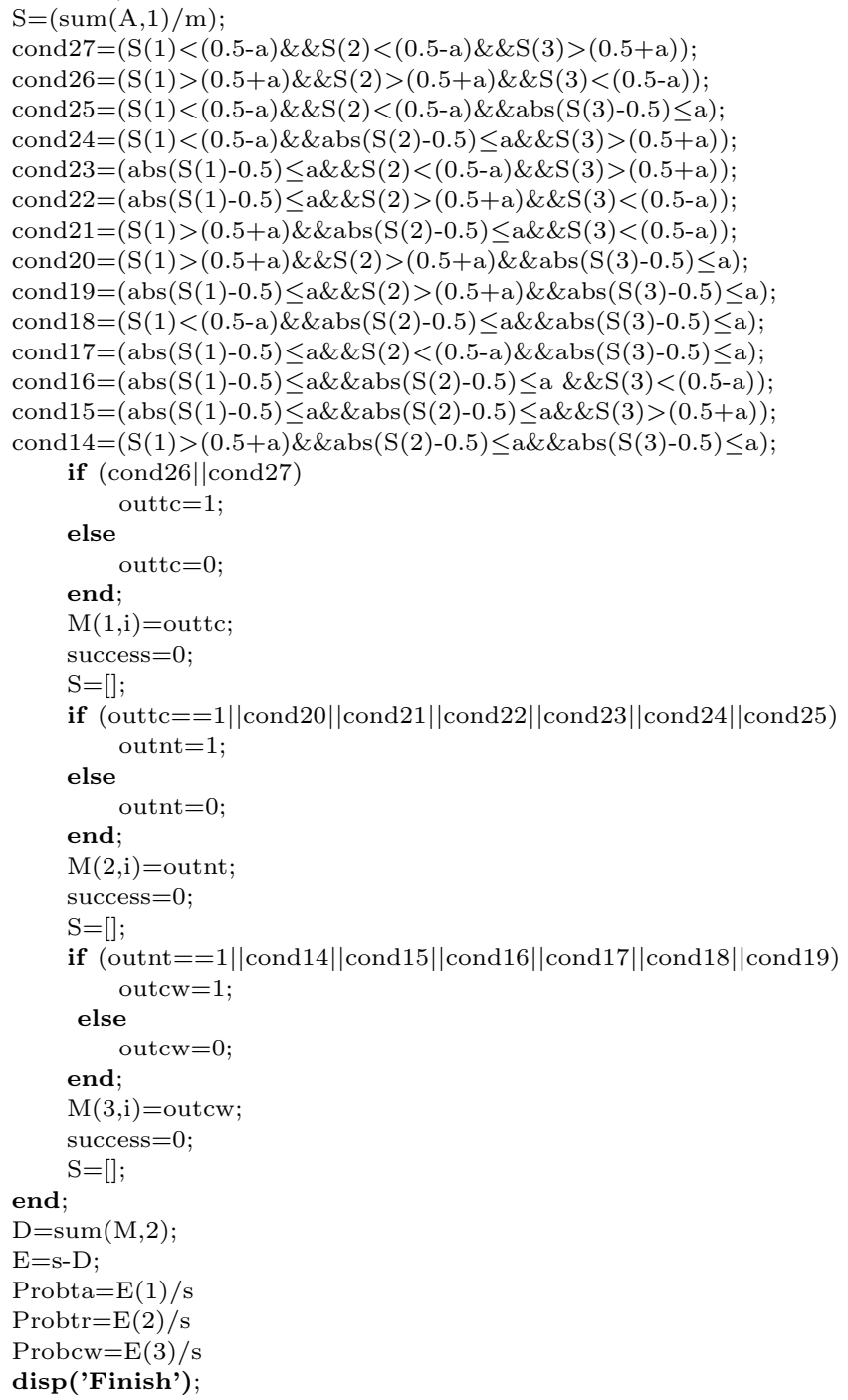

In the appendix, the simulated probabilities of consistent collective decisions under $M_{k}$ majorities are listed in tables. Main results are introduced in Section 4. 


\section{Results: Probabilities of consistent collective decisions under majorities based on difference in support}

From our results, it is obvious to show that the probability of having transitive weak preferences $R_{k}$, the probability of having transitive strict preferences $P_{k}$, and the probability of having triple-acyclic strict preferences $P_{k}$ are the same when $k=0$ regarding each type of $g$-transitivity. For instance, for $k=$ 0 and 0.5-transitivity, Table A.1, Table A.5, and Table A.9 show that this probability is equal to 0.8835 for $m=3$. Indeed, when $k=0$, the indifference in each collective preference is not allowed and the possible social outcomes in Table 1 are reduced to cases from 1 to 6 and the two cycles 26 and 27. As a consequence, the three probabilities are the same. However, when the threshold $k$ increases, Table 2 illustrates that the lower the consistency condition over the collective decision is, the higher the simulated probability of consistency is, regarding each type of $g$-transitivity. To illustrate this result, we first take into account the threshold $k=0.1$ for $m=1,000$. For 0.5 -transitive individual preferences, we deduce from Table 2 that the probability of having transitive weak preferences $R_{k}(0.8679)$ is smaller than the one of having transitive strict preferences $P_{k}(0.8680)$, which is in turn smaller than the probability of having triple-acyclic strict preferences $P_{k}(0.8733)$. This is true for the other types of $g$-transitivity (i.e. min-transitivity, am-transitivity, and max-transitivity). We also take into account the threshold $k=3$ in order to illustrate that this variation holds for other values of $k$. In addition, Table 2 illustrates that as well as the threshold $k$ increases the difference between the three probabilities increases. For instance, for $k=0.1$ and 0.5 -transitivity, the difference between the probability of having transitive weak preferences $R_{k}$ and the probability of having triple-acyclic strict preferences $P_{k}$ is equal to 0.0054 whereas this difference is given by 0.1512 for $k=3$.

Table 2 Simulated probabilities of consistent decisions with $m=1,000$.

\begin{tabular}{c|r|cccc}
\hline & & 0.5 -trans. & min-trans. & am-trans. & max-trans. \\
\hline \multirow{3}{*}{$k=0.1$} & $R_{k}$ transitive & 0.8679 & 0.9459 & 0.9657 & 0.9713 \\
& $P_{k}$ transitive & 0.8680 & 0.9460 & 0.9658 & 0.9714 \\
& $P_{k}$ triple-acyclic & 0.8733 & 0.9491 & 0.9685 & 0.9739 \\
\hline \multirow{3}{*}{$k=3$} & $R_{k}$ transitive & 0.7816 & 0.8802 & 0.9113 & 0.9184 \\
& $P_{k}$ transitive & 0.8267 & 0.9245 & 0.9536 & 0.9609 \\
& $P_{k}$ triple-acyclic & 0.9328 & 0.9827 & 0.9923 & 0.9945 \\
\hline
\end{tabular}

One of the main facts we can observe from the simulations is that, for a given number of voters $m$, when the threshold $k$ increases, we find that:

- The probability of having transitive weak preferences $R_{k}$ first decreases and then increases. 
- The probability of having transitive strict preferences $P_{k}$ first decreases and then increases.

- The probability of having triple-acyclic strict preferences $P_{k}$ increases. This is true for each value of the threshold $k$, even for small values. Indeed, when $k=0$ the probability of triple-acyclic $P_{k}$ is equal to $0.8835,0.9563$, 0.9751, and 0.9777 for 0.5-transitivity, min-transitivity, am-transitivity, and max-transitivity, respectively. However, even for a small value $k=0.001$, the probability to observe a triple-acyclic $P_{k}$ increases to $0.8838,0.9566$, 0.9753 , and 0.9778 .

- These three facts hold with independence of the assumed individual transitivity condition.

These results are shown by the probabilities of each table in the Appendix when $m \geq 100$. However, for small values of $m$ and the considered thresholds, these simulations only show the increase in the probability of transitive $R_{k}$ and transitive $P_{k}$. That seems to contradict above remarks about the behavior of the probabilities. In Table 3 we set $m=3$ and consider several values of $k$ to clarify that.

Table 3 The impact of the threshold $k$ on the probability of consistent outcomes for a given number of voters. The example of $m=3$.

\begin{tabular}{l|r|ccccc}
\hline & & $k=0$ & $k=0.1$ & $k=0.5$ & $k=1$ & $k=2$ \\
\hline \multirow{3}{*}{0.5 -trans. } & $R_{k}$ transitive & 0.8835 & 0.8339 & 0.6110 & 0.5438 & 0.8883 \\
& $P_{k}$ transitive & 0.8835 & 0.8497 & 0.8626 & 0.9587 & 0.9994 \\
& $P_{k}$ triple-acyclic & 0.8835 & 0.9216 & 0.9890 & 0.9997 & 1 \\
\hline \multirow{3}{*}{ min-trans. } & $R_{k}$ transitive & 0.9563 & 0.9222 & 0.7444 & 0.6551 & 0.8978 \\
& $P_{k}$ transitive & 0.9563 & 0.9383 & 0.9642 & 0.9950 & 1 \\
& $P_{k}$ triple-acyclic & 0.9563 & 0.9779 & 0.9997 & 1 & 1 \\
\hline \multirow{3}{*}{ am-trans. } & $R_{k}$ transitive & 0.9751 & 0.9476 & 0.7987 & 0.7036 & 0.8913 \\
& $P_{k}$ transitive & 0.9751 & 0.9629 & 0.9844 & 0.9988 & 1 \\
& $P_{k}$ triple-acyclic & 0.9751 & 0.9899 & 1.0000 & 1 & 1 \\
\hline \multirow{3}{*}{ max-trans. } & $R_{k}$ transitive & 0.9777 & 0.9518 & 0.8029 & 0.7102 & 0.9015 \\
& $P_{k}$ transitive & 0.9777 & 0.9679 & 0.9896 & 0.9993 & 1 \\
& $P_{k}$ triple-acyclic & 0.9777 & 0.9915 & 1.0000 & 1 & 1 \\
\hline
\end{tabular}

Going deeper in the probability of the transitive collective weak preference, we can not establish a clear relationship between the strength of the considered $g$-transitivity condition and that probability as we can see in Table 4 . For example, for a number of voters equal to 100, the required threshold for a simulated probability equal to 1 is 26.40 in the case of 0.5 -transitive reciprocal preference relations, 27.23 for min-transitive reciprocal preference relations, 27.90 for am-transitive ones and 25.47 in the case of max-transitive reciprocal preference relations. 
Table 4 Thresholds $k$ for simulated probabilities of $R_{k}$ being transitive equal to 1 .

\begin{tabular}{rcccc}
\hline & $m=3$ & $m=10$ & $m=100$ & $m=1,000$ \\
\hline $0.5-$ trans. & 2.97 & 7.95 & 26.40 & 84.83 \\
min-trans. & 2.96 & 8.32 & 27.23 & 95.32 \\
am-trans. & 2.97 & 7.48 & 27.90 & 83.01 \\
max-trans. & 2.98 & 7.56 & 25.47 & 80.00 \\
\hline
\end{tabular}

However, looking at having the probability of the strict preference being transitive equal to 1 (see Table 5 ), we can see that the higher the $g$-transitivity condition is, the lower the required thresholds $k$ are. We find an exception to that in the case of $m=100$, given that the threshold when regarding amtransitive reciprocal preference relations is 9.17 whereas the one considering max-transitive reciprocal preference relations equals 9.72 and therefore exceeds the previous one.

Table 5 Thresholds $k$ for simulated probabilities of $P_{k}$ being transitive equal to 1 .

\begin{tabular}{rcccc}
\hline & $m=3$ & $m=10$ & $m=100$ & $m=1,000$ \\
\hline 0.5 -trans. & 2.58 & 5.17 & 14.51 & 47.79 \\
min-trans. & 1.96 & 3.47 & 12.51 & 35.24 \\
am-trans. & 1.56 & 3.10 & 9.17 & 33.10 \\
max-trans. & 1.51 & 2.78 & 9.72 & 26.10 \\
\hline
\end{tabular}

Considering the strict preference relation, we find that the higher the $g$ transitive condition is, the lower the thresholds $k$ that provide a simulated probability of having triple-acyclic strict preferences equal to 1 are, with the exception of the case of max-transitive reciprocal preference relations (see Table 6).

Table 6 Thresholds $k$ for simulated probabilities of $P_{k}$ being triple-acyclic equal to 1 .

\begin{tabular}{rcccccc}
\hline & $m=3$ & $m=4$ & $m=5$ & $m=10$ & $m=100$ & $m=1,000$ \\
\hline 0.5 -trans. & 1.32 & 1.50 & 1.91 & 2.65 & 8.29 & 26.60 \\
min-trans. & 0.67 & 0.88 & 0.90 & 1.40 & 4.56 & 14.99 \\
am-trans. & 0.56 & 0.67 & 0.83 & 1.11 & 3.34 & 11.68 \\
max-trans. & 0.67 & 0.72 & 0.75 & 0.99 & 3.58 & 9.95 \\
\hline
\end{tabular}

Furthermore, as we can see in Table 7, regarding the value of the threshold $k$ that provides a simulated probability of the strict preference relation $P_{k}$ being transitive equal to 1 , the percentage $k / m$ decreases when the value of $m$ 
increases. For example, look at the case of max-transitive reciprocal preference relations. The percentage that the threshold represents over the number of voters is a $50, \widehat{3}$ percent in the case of $m=3$, a 27.8 percent for $m=10$, a 9.72 percent for $m=100$ and a 2.61 percent in the instance of $m=1,000$.

Table $7 \mathrm{k} / \mathrm{m}(\%)$ and $P_{k}$ transitive.

\begin{tabular}{rrlrlrrrr}
\hline & \multicolumn{2}{c}{$0.5-$ trans. } & \multicolumn{2}{c}{ min-trans. } & \multicolumn{2}{c}{ am-trans. } & \multicolumn{2}{c}{ max-trans. } \\
$m$ & $k$ & $k / m(\%)$ & $k$ & $k / m(\%)$ & $k$ & $k / m(\%)$ & $k$ & $k / m(\%)$ \\
\hline 3 & 2.58 & 86 & 1.96 & $65 . \widehat{3}$ & 1.56 & 52 & 1.51 & 50.3 \\
10 & 5.17 & 51.7 & 3.47 & 34.7 & 3.10 & 31 & 2.78 & 27.8 \\
100 & 14.51 & 14.51 & 12.51 & 12.51 & 9.17 & 9.17 & 9.72 & 9.72 \\
1,000 & 47.79 & 4.78 & 35.24 & 3.52 & 33.10 & 3.31 & 26.10 & 2.61 \\
\hline
\end{tabular}

Finally, considering the value of $k$ that provides a simulated probability of $P_{k}$ being triple-acyclic equal to 1 , the percentage $k / m$ decreases when the value of $m$ increases, considering min-transitive and am-transitive reciprocal preference relations. In Table 8, we have those percentages. As we can see, this is not the case for 0.5-transitive reciprocal preference relations when regarding $m=4$ and $m=5$ and max-transitive reciprocal preference relations when considering $m=5, m=100$ and $m=1,000$.

Table $8 \mathrm{k} / \mathrm{m}(\%)$ and $P_{k}$ triple-acyclic.

\begin{tabular}{rrrrrrrrc}
\hline & \multicolumn{2}{c}{0.5 -trans. } & \multicolumn{2}{c}{ min-trans. } & \multicolumn{2}{c}{ am-trans. } & \multicolumn{2}{c}{ max-trans. } \\
$m$ & $k$ & $k / m(\%)$ & $k$ & $k / m(\%)$ & $k$ & $k / m(\%)$ & $k$ & $k / m(\%)$ \\
\hline 3 & 1.32 & 44 & 0.67 & $22 . \widehat{3}$ & 0.56 & 18.6 & 0.67 & $22 . \widehat{3}$ \\
4 & 1.50 & 37.5 & 0.88 & 22 & 0.67 & 16.75 & 0.72 & 18 \\
5 & 1.91 & 38.2 & 0.90 & 18 & 0.83 & $16 . \widehat{6}$ & 0.75 & 0.15 \\
100 & 8.29 & 26.5 & 4.56 & 4.56 & 3.34 & 3.34 & 3.58 & 3.58 \\
1,000 & 26.60 & 2.66 & 14.99 & 1.499 & 11.68 & 1.168 & 9.95 & 0.995 \\
\hline
\end{tabular}

\section{Discussion}

Recently some analytical studies about the consistency of majorities based on difference in support have been developed. These theoretical results rely on the needed threshold to ensure transitive and triple-acyclic strict preferences for different types of transitive reciprocal preference relations.

On the one hand, the case of transitive strict preferences is studied in Llamazares et al (2013). The results can be summarized as follows:

1. The transitivity of the strict preference can not be ensured for any threshold of support $k$ less than $m-1$. 
2. The transitivity of the strict preference can not be ensured for any threshold of support $k$ less than $m$ if the reciprocal preference relations are less demanding than am-transitive ones.

3. The strict preference is transitive for any threshold of support such that $k \in$ $[m-1, m)$ if the reciprocal preference relations are at least am-transitive ones.

On the other hand, the case of triple-acyclic strict preferences is analysed in Llamazares and Pérez-Asurmendi (2015) with the following results:

1. The triple-acyclicity of the strict preference, in the case of 0.5 -transitive reciprocal preference relations, can be guaranteed if the threshold of support $k$ is located in $[\lfloor 2 m / 3\rfloor, m)$ where $\lfloor a\rfloor$ stands for the integer part of a.

2. The triple-acyclicity of the strict preference, in the case of min-transitive and max-transitive reciprocal preference relations, can be guaranteed if the threshold of support $k$ belongs to $[m / 3, m)$.

3. In the case of max-transitive reciprocal preference relations, it conjectures that strict preference relations are triple-acyclic if the threshold $k$ belongs to $[\lfloor 2 m / 3\rfloor / 2, m)$.

The probabilistic results setting here complement the above theoretical ones by the following reasons. First, thresholds with associated probabilities of consistent strict preferences equal to 1 are found for all the considered types of transitive reciprocal preference relations. Second, reasonable thresholds are required to certify the consistency of the strict preference with a probability value of 1 in those cases where theoretical results asked a very high threshold to guarantee such consistency. Third, the conjecture about the needed thresholds in the case of max-transitive reciprocal preference relations seems to be true.

Specifically, in the case of transitive strict preferences with 0.5 -transitive and min-transitive reciprocal preference relations, the probabilities achieve the value of 1 for the considered values of $m$ (see Table 9 ) whereas as it is said before, the theoretical result asserts that no threshold guarantees the transitivity of the strict preference for such types of reciprocal preference relations.

Table 9 Thresholds $k$ such that the simulated probability of $P_{k}$ being transitive is equal to 1 with 0.5 - and min-transitive reciprocal preference relations.

\begin{tabular}{rcccccc}
\hline & $m=3$ & $m=4$ & $m=5$ & $m=10$ & $m=100$ & $m=1,000$ \\
\hline$k(0.5$-trans.) & 2.58 & 2.97 & 3.24 & 5.17 & 14.51 & 47.79 \\
$k$ (min-trans.) & 1.96 & 2.49 & 2.61 & 3.47 & 12.51 & 35.24 \\
\hline
\end{tabular}

In the cases of am-transitive and max-transitive reciprocal preference relations, the thresholds that provide a probability value of transitive strict preference relations equal to 1 are lower than the ones that guarantee the transitivity of the strict preference in the theoretical framework. Table 10 shows 
the theoretical minimum threshold required, i. e. $m-1$, and the thresholds that provide a simulated probability value equal to 1 . To illustrate, look at the case of $m=1,000$. The theoretical result asserts that the threshold $k$ has to belong to $[999,1,000)$. By contrast, a probability value of 1 is achieved with a threshold of 33.10 in the case of am-transitive reciprocal preference relations and of 26.10 in the case of max-transitive reciprocal preference relations.

Table 10 Theoretical threshold $k$ vs. thresholds $k$ such that the simulated probability of $P_{k}$ being transitive is equal to 1 with am- and max-transitive reciprocal preference relations.

\begin{tabular}{rcccccc}
\hline & $m=3$ & $m=4$ & $m=5$ & $m=10$ & $m=100$ & $m=1,000$ \\
\hline$m-1$ & 2 & 3 & 4 & 9 & 99 & 999 \\
\hline$k$ (am-trans.) & 1.56 & 1.85 & 2.07 & 3.10 & 9.17 & 33.10 \\
$k$ (max-trans.) & 1.51 & 1.66 & 1.93 & 2.78 & 9.72 & 26.10 \\
\hline
\end{tabular}

In the case of triple-acyclic strict preferences, the thresholds to reach a probability value of 1 again are much lower than the ones required in the theoretical setting. Tables 11,12 and 13 illustrate that fact in the cases of 0.5 and min-transitive reciprocal preference relations. In these cases we set forth the minimum theoretical threshold required and the thresholds that provide a simulated probability value equal to 1 .

Table 11 Theoretical threshold $k$ vs. thresholds $k$ such that the simulated probability of $P_{k}$ being triple-acyclic is equal to 1 with 0.5 -transitive reciprocal preference relations.

\begin{tabular}{rcccccc}
\hline & $m=3$ & $m=4$ & $m=5$ & $m=10$ & $m=100$ & $m=1,000$ \\
\hline$\lfloor 2 m / 3\rfloor$ & 2 & 2 & 3 & 3 & 66 & 666 \\
$k$ & 1.32 & 1.50 & 1.91 & 2.65 & 8.29 & 26.60 \\
\hline
\end{tabular}

Table 12 Theoretical threshold $k$ vs. thresholds $k$ such that the simulated probability of $P_{k}$ being triple-acyclic is equal to 1 with min-transitive reciprocal preference relations.

\begin{tabular}{rlllllc}
\hline & $m=3$ & $m=4$ & $m=5$ & $m=10$ & $m=100$ & $m=1,000$ \\
\hline$m / 3$ & 1 & $1 . \widehat{3}$ & $1 . \widehat{6}$ & $3 . \widehat{3}$ & $33 . \widehat{3}$ & $333 . \widehat{3}$ \\
$k$ & 0.67 & 0.88 & 0.90 & 1.40 & 4.56 & 14.99 \\
\hline
\end{tabular}

The case of max-transitive reciprocal preference relations deserves a special attention. In Llamazares and Pérez-Asurmendi (2015), authors conjecture that the needed threshold to guarantee the triple-acyclicity of the collective 
strict preference when individuals are endowed with max-transitive reciprocal relations is lower or equal ${ }^{3}$ than in the case of individual min-transitive reciprocal relations. Unfortunately, they could not provide a formal proof for that assertation. Comparing the thresholds $k$ such that the simulated probability of $P_{k}$ is triple-acylic with a probability of 1 from Tables 12 and 13, we can corroborate that conjecture. The thresholds in the case of max-transitive reciprocal preference relations are smaller or equal than the ones in min-transitive case.

Table 13 Theoretical threshold $k$ vs. thresholds $k$ such that the simulated probability of $P_{k}$ being triple-acyclic is equal to 1 with max-transitive reciprocal preference relations.

\begin{tabular}{rcccccc}
\hline & $m=3$ & $m=4$ & $m=5$ & $m=10$ & $m=100$ & $m=1,000$ \\
\hline$[\lfloor 2 m / 3\rfloor / 2, m)$ & 1 & 1 & 1.5 & 3 & 33 & 333 \\
$k$ & 0.67 & 0.72 & 0.75 & 0.99 & 3.58 & 9.95 \\
\hline
\end{tabular}

A quite natural question is to wonder if the consideration of reciprocal preference relations has an impact on the probability of consistent decisions when compared with the case of crisp preferences, i.e. when voters only declare if they prefer an alternative to another one or if they are indifferent between both alternatives.

To highlight this idea, we consider two cases: First, we focus on voters who show linear preference orderings on the candidates. In other words, $r_{i j}^{p} \in\{0,1\}$ for each pair of alternatives $x_{i}$ and $x_{j}$ and each voter $p$. Second, we also consider the case where individuals are endowed with weak preference orderings and declare their preferences by means of values $r_{i j}^{p} \in\{0,0.5,1\}$. If $r_{i j}^{p}=0.5$, individual $p$ is indifferent between both alternatives. Notice that in the first case, these majorities are equivalent to supermajorities (see for a formal proof of that Diss and Pérez-Asurmendi (2015)); in the second case, these voting rules are majorities based on difference of votes.

For that aim, we find the thresholds which guarantee a probability equal to 1 of $P_{k}$ being triple-acyclic (Table 14), $P_{k}$ being transitive (Table 15), and $R_{k}$ being transitive (Table 16) taking into account individual linear and weak orderings and compare them with the corresponding ones when considering individual reciprocal preference relations.

3 Notice that $m / 3=\lfloor(2 \cdot 100) / 3\rfloor / 2$ when $m$ is multiple of three. 
Table 14 Crisp preferences versus reciprocal preferences: Thresholds $k$ such that the simulated probability of $P_{k}$ being triple-acyclic is equal to 1 .

\begin{tabular}{l|cc|cccc}
\hline \multirow{2}{*}{$m \downarrow$} & \multicolumn{2}{|c|}{ Crisp preferences } & \multicolumn{4}{c}{ Reciprocal preferences } \\
\cline { 2 - 6 } & $r_{i j}^{p} \in\{0,1\}$ & $r_{i j}^{p} \in\{0,0.5,1\}$ & 0.5 -trans. & min-trans. & am-trans. & max-trans. \\
\hline 3 & 1 & 1 & 1.32 & 0.67 & 0.56 & 0.67 \\
4 & 0 & 1 & 1.50 & 0.88 & 0.67 & 0.72 \\
5 & 1 & 1 & 1.91 & 0.90 & 0.83 & 0.75 \\
10 & 2 & 2 & 2.65 & 1.40 & 1.11 & 0.99 \\
100 & 10 & 8 & 8.29 & 4.56 & 3.34 & 3.58 \\
1000 & 36 & 30 & 26.60 & 14.99 & 11.68 & 9.95 \\
\hline
\end{tabular}

Table 15 Crisp preferences versus reciprocal preferences: Thresholds $k$ such that the simulated probability of $P_{k}$ being transitive is equal to 1 .

\begin{tabular}{l|cc|cccc}
\hline \multirow{2}{*}{$m \downarrow$} & \multicolumn{2}{|c|}{ Crisp preferences } & \multicolumn{3}{c}{ Reciprocal preferences } \\
\cline { 2 - 6 } & $r_{i j}^{p} \in\{0,1\}$ & $r_{i j}^{p} \in\{0,0.5,1\}$ & 0.5 -trans. & min-trans. & am-trans. & max-trans. \\
\hline 3 & 1 & 1 & 2.58 & 1.96 & 1.56 & 1.51 \\
4 & 2 & 2 & 2.97 & 2.49 & 1.85 & 1.66 \\
5 & 3 & 3 & 3.24 & 2.61 & 2.07 & 1.93 \\
10 & 6 & 7 & 5.17 & 3.47 & 3.10 & 2.78 \\
100 & 26 & 21 & 14.51 & 12.51 & 9.17 & 9.72 \\
1000 & 78 & 89 & 47.79 & 35.24 & 33.10 & 26.10 \\
\hline
\end{tabular}

Table 16 Crisp preferences versus reciprocal preferences: Thresholds $k$ such that the simulated probability of $R_{k}$ being transitive is equal to 1 .

\begin{tabular}{l|cc|cccc}
\hline \multirow{2}{*}{$m \downarrow$} & \multicolumn{2}{|c|}{ Crisp preferences } & \multicolumn{4}{c}{ Reciprocal preferences } \\
\cline { 2 - 6 } & $r_{i j}^{p} \in\{0,1\}$ & $r_{i j}^{p} \in\{0,0.5,1\}$ & 0.5 -trans. & min-trans. & am-trans. & max-trans. \\
\hline 3 & - & - & 2.97 & 2.96 & 2.97 & 2.98 \\
4 & - & - & 3.81 & 3.84 & 3.91 & 3.84 \\
5 & - & - & 4.70 & 4.80 & 4.91 & 4.72 \\
10 & - & - & 7.95 & 8.32 & 7.48 & 7.56 \\
100 & 48 & 43 & 26.40 & 27.23 & 27.90 & 25.47 \\
1000 & 147 & 172 & 84.83 & 95.32 & 83.01 & 80.00 \\
\hline
\end{tabular}

We can deduce the following facts:

- For big electorates, required thresholds are lower when considering reciprocal preference relations than in the case of crisp preferences with independence of the analysed collective consistent decision (see the case of $m=1,000$ in Tables 14, 15, and 16). 
- In the case of the probability of $R_{k}$ being transitive equal to 1 (Table 16), we find thresholds to reach that probability when considering reciprocal preference relations for all the considered number of individuals whilst in the case of crisp preferences we cannot provide some of them. Notice that, even for a threshold equal to $k=m-1$, the probability of transitive $R_{k}$ is non null when considering crisp preferences. For instance, for $m=10$ and a threshold $k=9$, the probability of transitive $R_{k}$ is equal to 0.9944 and 0.9993 for $r_{i j}^{p} \in\{0,1\}$ and $r_{i j}^{p} \in\{0,0.5,1\}$, respectively.

- In the case of the probability of $P_{k}$ being triple-acyclic equal to 1 (Table 14), we find lower thresholds for min-transitive, am-transitive and max-transitive reciprocal preference relations than for crisp preferences.

- The case of the probability of $P_{k}$ being transitive equal to 1 (Table 15) is more complex to analyse. On the one hand, the thresholds are lower when considering crisp preferences and $m=3$. On the other hand, reciprocal preferences provide lower thresholds for bigger electorates than that. Specifically, greater than $m=3$ when considering am-transitive and maxtransitive reciprocal preferences relations, greater than $m=4$ in the case of min-transitive reciprocal preference relations and greater than $m=10$ when regarding 0.5-transitive reciprocal preference relations.

Therefore, we can ensure that considering reciprocal preference relations has an unequivocal effect on the needed thresholds to reach a probability of collective consistent decisions equal to 1 when considering big electorates.

Finally, we set forth the probabilities of consistent collective decisions with crisp preferences and the ones with reciprocal preferences for a given number of voters $m$ and a given threshold $k$. Now our goal is to know if the likelihood of consistent collective decisions is higher or smaller when the voters show their preferences reciprocally than in the case where they are endowed with linear or weak orderings. In Table 17, we set the probabilities when $m=3$ and $m=1,000$ with the aim of distinguishing between the cases of small and big electorates. 
Table 17 The impact of the individual type of preferences in the probability. The examples of $m=3$ and $m=1,000$.

\begin{tabular}{|c|c|c|c|c|c|c|c|}
\hline & & \multicolumn{3}{|c|}{$m=3$} & \multicolumn{3}{|c|}{$m=1,000$} \\
\hline & & $k=0$ & $k=1$ & $k=2$ & $k=3$ & $k=4$ & $k=10$ \\
\hline$r_{i j}^{p} \in\{0,1\}$ & $\begin{array}{r}R_{k} \text { transitive } \\
P_{k} \text { transitive } \\
P_{k} \text { triple-acyclic }\end{array}$ & $\begin{array}{l}0.9511 \\
0.9511 \\
0.9511 \\
\end{array}$ & $\begin{array}{c}0.6772 \\
1 \\
1 \\
\end{array}$ & $\begin{array}{c}0.6908 \\
1 \\
1 \\
\end{array}$ & $\begin{array}{l}0.8739 \\
0.8907 \\
0.9492 \\
\end{array}$ & $\begin{array}{l}0.9004 \\
0.9160 \\
0.9643 \\
\end{array}$ & $\begin{array}{l}0.8047 \\
0.9137 \\
0.9897 \\
\end{array}$ \\
\hline$r_{i j}^{p} \in\{0,0.5,1\}$ & $\begin{array}{r}R_{k} \text { transitive } \\
P_{k} \text { transitive } \\
P_{k} \text { triple-acyclic }\end{array}$ & $\begin{array}{l}0.8736 \\
0.9599 \\
0.9944 \\
\end{array}$ & $\begin{array}{c}0.6943 \\
1 \\
1\end{array}$ & $\begin{array}{c}0.7827 \\
1 \\
1 \\
\end{array}$ & $\begin{array}{l}0.9399 \\
0.9442 \\
0.9727 \\
\end{array}$ & $\begin{array}{l}0.9339 \\
0.9411 \\
0.9761 \\
\end{array}$ & $\begin{array}{l}0.8893 \\
0.9272 \\
0.9910 \\
\end{array}$ \\
\hline 0.5 -trans. & $\begin{array}{r}R_{k} \text { transitive } \\
P_{k} \text { transitive } \\
P_{k} \text { triple-acyclic } \\
\end{array}$ & $\begin{array}{l}0.8835 \\
0.8835 \\
0.8835 \\
\end{array}$ & $\begin{array}{l}0.5438 \\
0.9587 \\
0.9997 \\
\end{array}$ & $\begin{array}{c}0.8883 \\
0.9994 \\
1\end{array}$ & $\begin{array}{l}0.7816 \\
0.8267 \\
0.9328\end{array}$ & $\begin{array}{l}0.7479 \\
0.8241 \\
0.9477 \\
\end{array}$ & $\begin{array}{l}0.5794 \\
0.8767 \\
0.9905 \\
\end{array}$ \\
\hline $\min -t$ & $\begin{array}{r}R_{k} \text { transitive } \\
P_{k} \text { transitive } \\
P_{k} \text { triple-acyclic } \\
\end{array}$ & $\begin{array}{l}0.9563 \\
0.9563 \\
0.9563 \\
\end{array}$ & $\begin{array}{c}0.6551 \\
0.9950 \\
1 \\
\end{array}$ & $\begin{array}{c}0.8978 \\
1 \\
1 \\
\end{array}$ & $\begin{array}{l}0.8802 \\
0.9245 \\
0.9827 \\
\end{array}$ & $\begin{array}{l}0.8534 \\
0.9262 \\
0.9886\end{array}$ & $\begin{array}{l}0.7092 \\
0.9659 \\
0.9996 \\
\end{array}$ \\
\hline am-trans. & $\begin{array}{r}R_{k} \text { transitive } \\
P_{k} \text { transitive } \\
P_{k} \text { triple-acyclic }\end{array}$ & $\begin{array}{l}0.9751 \\
0.9751 \\
0.9751 \\
\end{array}$ & $\begin{array}{c}0.7036 \\
0.9988 \\
1 \\
\end{array}$ & $\begin{array}{c}0.8913 \\
1 \\
1 \\
\end{array}$ & $\begin{array}{l}0.9113 \\
0.9536 \\
0.9923 \\
\end{array}$ & $\begin{array}{l}0.8883 \\
0.9572 \\
0.9959 \\
\end{array}$ & $\begin{array}{l}0.7640 \\
0.9857 \\
1.0000 \\
\end{array}$ \\
\hline max-trans. & $\begin{array}{r}R_{k} \text { transitive } \\
P_{k} \text { transitive } \\
P_{k} \text { triple-acyclic }\end{array}$ & $\begin{array}{l}0.9777 \\
0.9777 \\
0.9777\end{array}$ & $\begin{array}{c}0.7102 \\
0.9993 \\
1\end{array}$ & $\begin{array}{c}0.9015 \\
1 \\
1\end{array}$ & $\begin{array}{l}0.9184 \\
0.9609 \\
0.9945\end{array}$ & $\begin{array}{l}0.8971 \\
0.9657 \\
0.9970\end{array}$ & $\begin{array}{c}0.7726 \\
0.9904 \\
1\end{array}$ \\
\hline
\end{tabular}

For a small electorate $(m=3)$ we have found the following:

- For $k=0$, the probabilities of transitive $R_{k}$ are greater when considering min-transitive, am-transitive and max-transitive reciprocal preference relations than when considering crisp and 0.5-transitive reciprocal preferences. The probabilities of transitive $P_{k}$ are also greater bearing in mind am-transitive and max-transitive reciprocal preference relations than any other considering preferences. Nevertheless, the probability of triple-acyclic $P_{k}$ is greater in the case of weak orderings than in the other possible cases.

- For $k=1$, the probabilities of transitive $R_{k}$ are greater when regarding amtransitive and max-transitive reciprocal preference relations than when considering crisp preferences and these last ones are also greater than when taking into account min-transitive and 0.5-transitive reciprocal preference relations. The probabilities of transitive $P_{k}$ are greater when regarding crisp preferences than when considering reciprocal preference relations. In the case of the probabilities of triple-acyclic $P_{k}$, crisp preferences and reciprocal preference relations provide same probabilities with the exception of the case of 0.5 -reciprocal preference relations attached with a lower probability.

- For $k=2$, the probabilities of consistent outcomes are greater or equal when considering reciprocal preference relations than when regarding crisp preferences with the exception of the probability of transitive $P_{k}$ when having 0.5 reciprocal preference relations.

For a big electorate $(m=1,000)$ we have the following: 
- For $k=3$ the lowest probabilities of consistent outcomes are reached when considering 0.5 -transitive reciprocal preference relations. The probabilities of transitive $R_{k}$ are greater when considering weak orderings than maxtransitive, am-transitive, min-transitive reciprocal preference relations and those ones are also greater than when regarding linear orderings. The probabilities of transitive $P_{k}$ are greater when considering am-transitive and max-transitive reciprocal relations than when regarding weak orderings. These last ones are greater than when considering min-transitive reciprocal relations which in turn are also greater than when regarding linear orderings. In the case of the probabilities of triple-acylcic $P_{k}$, the greatest ones corresponds to max-transitive, am-transitive and min-transitive reciprocal preference relations followed by the ones attached to crisp preferences.

- For $k=4$ the probabilities of transitive $R_{k}$ are greater when considering crisp than reciprocal preference relations. The probabilities of transitive and triple-acyclic $P_{k}$ behave in the same way than in the case of $k=3$ analysed above.

- For $k=10$ the probabilities of transitive $R_{k}$ are greater when considering crisp than reciprocal preference relations. The probabilities of transitive $P_{k}$ are greater when considering max-transitive, am-transitive and mintransitive reciprocal preference relations than when considering crisp preferences. The lowest probabilities are reached when regarding 0.5 -transitive reciprocal preference relations. The probabilities of triple-acylcic $P_{k}$ are greater when considering reciprocal preference relations than when regarding crisp preferences.

Therefore, we can not draw a clear conclusion about the relationship between the probabilities and the considered type of individual preferences. For instance, 0.5-transitive reciprocal preference relations give rise to the lowest probabilities of consistent outcomes except in the cases of triple-acyclic $P_{k}$ with $k=2$ for small electorates and with $k=10$ for big electorates, respectively.

For big electorates weak orderings provide greater probabilities of transitive $R_{k}$ than the remaining considered individual preferences. Instead, in the case of small electorates, am-transitive and max-transitive reciprocal preference relations promote the greatest probabilities.

Analysing the case of the probabilities of transitive $P_{k}$ considering big electorates, the greatest ones are reached when regarding max-transitive and am-transitive reciprocal preference relations. That is also the case for $m=3$ when considering $k=0$ but not for the other considered values of $k$ (notice that in the case of $k=1$ crisp preferences give rise to the highest probabilities and in the case of $k=2$ the probabilities are the same for all the considered individual preferences with the exception of 0.5 -transitive reciprocal preference relations).

Finally, when regarding the probabilities of triple-acyclic $P_{k}$ we found that the greatest probabilities are attached to weak orderings when $m=3$ whereas 
to max-transitive, am-transitive and min-transitive reciprocal preference relations when $m=1,000$.

\section{Conclusion}

In this paper, we estimate through simulations the probabilities of consistent preferences under majorities based on difference in support: the probability of having transitive weak preferences $R_{k}$, the probability of having transitive strict preferences $P_{k}$, and the probability of having triple-acyclic strict preferences $P_{k}$. We start our simulations by assuming four types of transitivity conditions for individual reciprocal preferences: 0.5-transitivity, min-transitivity, am-transitivity, and max-transitivity.

This paper contains new contributions to the literature of reciprocal preferences. First, we have found the thresholds which guarantee that the probability of consistent outcomes is equal to 1 . These thresholds are much lower than the ones required in the theoretical setting. In fact, our results show a dramatic difference between these two thresholds: For instance, in some cases, the simulated threshold represents less than a $3 \%$ of the total number of voters whereas the theoretical one reaches the $67 \%$. Given that the needed thresholds to reach consistent decisions with probability equal to 1 are not too demanding, the implementation of majorities based in difference of support have sense whenever the flexibility attached to individual preferences by the consideration of reciprocal preference relations and the consistency of the collective decision is a concern for the policy maker or for the society as a whole. Second, we have set forth the impact of the threshold $k$ on the probability of consistent outcomes for a given number of voters. The probability of having transitive weak preferences $R_{k}$ as well as the probability of having transitive strict preferences $P_{k}$ exhibit a similar behaviour: for the lowest values of the threshold $k$, it decreases as the value of the threshold $k$ increases whereas for higher values of $k$, it increases with the value of the threshold $k$. However, the probability of having triple-acyclic strict preferences $P_{k}$ increases when the value of the threshold $k$ does, for any number of voters. Third, we have compared the needed thresholds to reach a probability of collective consistent decisions equal to 1 regarding reciprocal preference relations with the ones considering crisp preferences and concluded that the thresholds are significantly lower in the case of reciprocal preference relations than in the case of crisp preferences. Finally, we have studied if the consideration of reciprocal preference relations instead of crisp preferences has an impact in the probability of collective consistent decisions. We have found that the probability depends on the concrete considered individual preference relation and on the size of the electorate. 
Appendix

Table A.1 Probabilities of transitive $R_{k}$ for 0.5 -transitive reciprocal preference relations.

\begin{tabular}{c|lllllll}
\hline $\begin{array}{l}m \rightarrow \\
k \downarrow\end{array}$ & 3 & 4 & 5 & 10 & 100 & 1,000 & 100,000 \\
\hline 0 & 0.8835 & 0.8781 & 0.8763 & 0.8751 & 0.8706 & 0.8705 & 0.8728 \\
2.97 & 1 & 0.9836 & 0.9485 & 0.7630 & 0.5927 & 0.7826 & 0.8655 \\
3.81 & & 1 & 0.9964 & 0.9065 & 0.5481 & 0.7544 & 0.8633 \\
4.70 & & & 1 & 0.9748 & 0.5312 & 0.7248 & 0.8609 \\
7.95 & & & & 1 & 0.6729 & 0.6266 & 0.8518 \\
26.40 & & & & & 1 & 0.7012 & 0.7954 \\
84.83 & & & & & & 1 & 0.6156 \\
95.32 & & & & & & & 0.5913 \\
\hline
\end{tabular}

Table A.2 Probabilities of transitive $R_{k}$ for min-transitive reciprocal preference relations.

\begin{tabular}{c|lllllll}
\hline $\begin{array}{l}m \rightarrow \\
k \downarrow\end{array}$ & 3 & 4 & 5 & 10 & 100 & 1,000 & 100,000 \\
\hline 0 & 0.9563 & 0.9513 & 0.9505 & 0.9487 & 0.9468 & 0.9476 & 0.9455 \\
2.96 & 1 & 0.9831 & 0.9528 & 0.8015 & 0.7220 & 0.8814 & 0.9400 \\
3.84 & & 1 & 0.9966 & 0.9205 & 0.6776 & 0.8576 & 0.9383 \\
4.80 & & & 1 & 0.9809 & 0.6552 & 0.8307 & 0.9366 \\
8.32 & & & & 1 & 0.7559 & 0.7432 & 0.9291 \\
27.23 & & & & & 1 & 0.7702 & 0.8831 \\
95.32 & & & & & & 1 & 0.7189 \\
\hline
\end{tabular}

Table A.3 Probabilities of transitive $R_{k}$ for am-transitive reciprocal preference relations.

\begin{tabular}{c|lllllll}
\hline $\begin{array}{l}m \rightarrow \\
k \downarrow\end{array}$ & 3 & 4 & 5 & 10 & 100 & 1,000 & 100,000 \\
\hline 0 & 0.9751 & 0.9710 & 0.9712 & 0.9694 & 0.9676 & 0.9670 & 0.9692 \\
2.97 & 1 & 0.9812 & 0.9492 & 0.8087 & 0.7738 & 0.9117 & 0.9650 \\
3.91 & & 1 & 0.9971 & 0.9225 & 0.7295 & 0.8904 & 0.9636 \\
4.91 & & & 1 & 0.9813 & 0.7043 & 0.8683 & 0.9622 \\
7.48 & & & & 1 & 0.7370 & 0.8112 & 0.9582 \\
27.90 & & & & & 1 & 0.7873 & 0.9180 \\
83.01 & & & & & & 1 & 0.7967 \\
95.32 & & & & & & & 0.7731 \\
\hline
\end{tabular}


Table A.4 Probabilities of transitive $R_{k}$ for $\max$-transitive reciprocal preference relations.

\begin{tabular}{c|lllllll}
\hline $\begin{array}{l}m \rightarrow \\
k \downarrow\end{array}$ & 3 & 4 & 5 & 10 & 100 & 1,000 & 100,000 \\
\hline 0 & 0.9777 & 0.9766 & 0.9751 & 0.9741 & 0.9727 & 0.9726 & 0.9744 \\
2.98 & 1 & 0.9842 & 0.9561 & 0.8219 & 0.7867 & 0.9189 & 0.9708 \\
3.84 & & 1 & 0.9964 & 0.9241 & 0.7450 & 0.9003 & 0.9696 \\
4.72 & & & 1 & 0.9782 & 0.7188 & 0.8805 & 0.9681 \\
7.56 & & & & 1 & 0.7495 & 0.8190 & 0.9637 \\
25.47 & & & & & 1 & 0.7720 & 0.9298 \\
80.00 & & & & & & 1 & 0.8030 \\
95.32 & & & & & & & 0.7744 \\
\hline
\end{tabular}

Table A.5 Probabilities of transitive $P_{k}$ for 0.5 -transitive reciprocal preference relations.

\begin{tabular}{c|lllllll}
\hline $\begin{array}{l}m \rightarrow \\
k \downarrow\end{array}$ & 3 & 4 & 5 & 10 & 100 & 1,000 & 100,000 \\
\hline 0 & 0.8835 & 0.8781 & 0.8763 & 0.8751 & 0.8706 & 0.8705 & 0.8728 \\
2.58 & 1 & 0.9999 & 0.9995 & 0.9916 & 0.8512 & 0.8287 & 0.8668 \\
2.97 & & 1 & 1.0000 & 0.9970 & 0.8661 & 0.8268 & 0.8659 \\
3.24 & & & 1 & 0.9985 & 0.8759 & 0.8257 & 0.8654 \\
5.17 & & & & 1 & 0.9465 & 0.8267 & 0.8611 \\
14.51 & & & & & 1 & 0.9295 & 0.8440 \\
47.79 & & & & & & 1 & 0.8293 \\
\hline
\end{tabular}

Table A.6 Probabilities of transitive $P_{k}$ for min-transitive reciprocal preference relations.

\begin{tabular}{|c|c|c|c|c|c|c|c|}
\hline $\begin{array}{l}m \rightarrow \\
k \downarrow\end{array}$ & 3 & 4 & 5 & 10 & 100 & 1,000 & 100,000 \\
\hline 0 & 0.9563 & 0.9513 & 0.9505 & 0.9487 & 0.9468 & 0.9476 & 0.9455 \\
\hline 1.96 & 1 & 0.9999 & 0.9997 & 0.9968 & 0.9377 & 0.9271 & 0.9421 \\
\hline 2.49 & & 1 & 1.0000 & 0.9993 & 0.9514 & 0.9255 & 0.9414 \\
\hline 2.61 & & & 1 & 0.9995 & 0.9533 & 0.9254 & 0.9413 \\
\hline 3.47 & & & & 1 & 0.9704 & 0.9248 & 0.9398 \\
\hline 12.51 & & & & & 1 & 0.9793 & 0.9291 \\
\hline 35.24 & & & & & & 1 & 0.9251 \\
\hline 47.79 & & & & & & & 0.9311 \\
\hline
\end{tabular}


Table A.7 Probabilities of transitive $P_{k}$ for am-transitive reciprocal preference relations.

\begin{tabular}{c|lllllll}
\hline $\begin{array}{l}m \rightarrow \\
k \downarrow\end{array}$ & 3 & 4 & 5 & 10 & 100 & 1,000 & 100,000 \\
\hline 0 & 0.9751 & 0.9710 & 0.9712 & 0.9694 & 0.9676 & 0.9670 & 0.9692 \\
1.56 & 1 & 0.9999 & 0.9997 & 0.9973 & 0.9605 & 0.9551 & 0.9672 \\
1.85 & & 1 & 1.0000 & 0.9990 & 0.9655 & 0.9542 & 0.9668 \\
2.07 & & & 1 & 0.9995 & 0.9697 & 0.9538 & 0.9665 \\
3.10 & & & & 1 & 0.9845 & 0.9538 & 0.9653 \\
9.17 & & & & & 1 & 0.9828 & 0.9600 \\
33.10 & & & & & & 1 & 0.9570 \\
47.79 & & & & & & & 0.9629 \\
\hline
\end{tabular}

Table A.8 Probabilities of transitive $P_{k}$ for max-transitive reciprocal preference relations.

\begin{tabular}{c|lllllll}
\hline $\begin{array}{l}m \rightarrow \\
k \downarrow\end{array}$ & 3 & 4 & 5 & 10 & 100 & 1,000 & 100,000 \\
\hline 0 & 0.9777 & 0.9766 & 0.9751 & 0.9741 & 0.9727 & 0.9726 & 0.9744 \\
1.51 & 1 & 1.0000 & 0.9999 & 0.9981 & 0.9689 & 0.9619 & 0.9727 \\
1.66 & & 1 & 0.9999 & 0.9989 & 0.9712 & 0.9614 & 0.9725 \\
1.93 & & & 1 & 0.9996 & 0.9758 & 0.9608 & 0.9723 \\
2.78 & & & & 1 & 0.9870 & 0.9607 & 0.9715 \\
9.72 & & & & & 1 & 0.9897 & 0.9662 \\
26.10 & & & & & & 1 & 0.9626 \\
47.79 & & & & & & & 0.9690 \\
\hline
\end{tabular}

Table A.9 Probabilities of triple-acyclic $P_{k}$ for 0.5 -transitive reciprocal preference relations.

\begin{tabular}{c|lllllll}
\hline $\begin{array}{l}m \rightarrow \\
k \downarrow\end{array}$ & 3 & 4 & 5 & 10 & 100 & 1,000 & 100,000 \\
\hline 0 & 0.8835 & 0.8781 & 0.8763 & 0.8751 & 0.8706 & 0.8705 & 0.8728 \\
1.32 & 1 & 0.9999 & 0.9998 & 0.9967 & 0.9479 & 0.9013 & 0.8763 \\
1.50 & & 1 & 1.0000 & 0.9983 & 0.9546 & 0.9052 & 0.8768 \\
1.91 & & & 1 & 0.9998 & 0.9681 & 0.9135 & 0.8778 \\
2.65 & & & & 1 & 0.9837 & 0.9270 & 0.8794 \\
8.29 & & & & & 1 & 0.9834 & 0.8926 \\
26.60 & & & & & & 1 & 0.9291 \\
\hline
\end{tabular}


Table A.10 Probabilities of triple-acyclic $P_{k}$ for min-transitive reciprocal preference relations.

\begin{tabular}{c|lllllll}
\hline $\begin{array}{l}m \rightarrow \\
k \downarrow\end{array}$ & 3 & 4 & 5 & 10 & 100 & 1,000 & 100,000 \\
\hline 0 & 0.9563 & 0.9513 & 0.9505 & 0.9487 & 0.9468 & 0.9476 & 0.9455 \\
0.67 & 1 & 0.9998 & 0.9997 & 0.9971 & 0.9812 & 0.9583 & 0.9467 \\
0.88 & & 1 & 1.0000 & 0.9993 & 0.9817 & 0.9612 & 0.9471 \\
0.90 & & & 1 & 0.9993 & 0.9906 & 0.9614 & 0.9472 \\
1.40 & & & & 1 & 0.9906 & 0.9675 & 0.9481 \\
4.56 & & & & & 1 & 0.9913 & 0.9540 \\
14.99 & & & & & & 1 & 0.9687 \\
26.60 & & & & & & & 0.9799 \\
\hline
\end{tabular}

Table A.11 Probabilities of triple-acyclic $P_{k}$ for am-transitive reciprocal preference relations.

\begin{tabular}{c|lllllll}
\hline $\begin{array}{l}m \rightarrow \\
k \downarrow\end{array}$ & 3 & 4 & 5 & 10 & 100 & 1,000 & 100,000 \\
\hline 0 & 0.9751 & 0.9710 & 0.9712 & 0.9694 & 0.9676 & 0.9670 & 0.9692 \\
0.56 & 1 & 1.0000 & 0.9998 & 0.9986 & 0.9854 & 0.9743 & 0.9698 \\
0.67 & & 1 & 1.0000 & 0.9995 & 0.9876 & 0.9758 & 0.9700 \\
0.83 & & & 1 & 0.9999 & 0.9905 & 0.9775 & 0.9702 \\
1.11 & & & & 1 & 0.9941 & 0.9804 & 0.9706 \\
3.34 & & & & & 1 & 0.9936 & 0.9730 \\
11.68 & & & & & & 1 & 0.9811 \\
26.60 & & & & & & & 0.9911 \\
\hline
\end{tabular}

Table A.12 Probabilities of triple-acyclic $P_{k}$ for max-transitive reciprocal preference relations.

\begin{tabular}{c|lllllll}
\hline $\begin{array}{l}m \rightarrow \\
k \downarrow\end{array}$ & 3 & 4 & 5 & 10 & 100 & 1,000 & 100,000 \\
\hline 0 & 0.9777 & 0.9766 & 0.9751 & 0.9741 & 0.9727 & 0.9726 & 0.9744 \\
0.67 & 1 & 1.0000 & 0.9999 & 0.9998 & 0.9909 & 0.9804 & 0.9753 \\
0.72 & & 1 & 1.0000 & 0.9999 & 0.9918 & 0.9808 & 0.9753 \\
0.75 & & & 1 & 0.9999 & 0.9922 & 0.9811 & 0.9754 \\
0.99 & & & & 1 & 0.9951 & 0.9834 & 0.9758 \\
3.58 & & & & & 1 & 0.9963 & 0.9786 \\
9.95 & & & & & & 1 & 0.9845 \\
26.60 & & & & & & & 0.9943 \\
\hline
\end{tabular}




\section{References}

Balasko Y, Crès H (1997) The probability of Condorcet cycles and super majority rules. J Econ Theory 75:237-270

Campbell CD, Tullock G (1965) A measure of the importance of cyclical majorities. Econ J 75(300):853-857

Cervone DP, Gehrlein WV, Zwicker WS (2005) Which scoring rule maximizes Condorcet efficiency under IAC? Theor Decis 58(2):145-185

Condorcet Md (1785) Essai sur l'Application de l'Analyse à la Probabilité des Décisions Rendues à la Pluralité des Voix. Imprimerie Royale, Paris

Cook WD and Kress M (1985) Ordinal Ranking with Intensity of Preference. Manage Sci $31: 26-32$

Dasgupta M, Deb R (1996) Transitivity and fuzzy preferences. Soc Choice Welfare 13(3):305318

DeMeyer F, Plott C (1970) The probability of a cyclical majority. Econometrica 38(2):345354

Diss M, Pérez-Asurmendi P (2015) Consistent collective decisions under Majorities based on difference of votes, Theory Dec, Forthcoming

Dubois D, Prade H (1980) Fuzzy Sets and Systems: Theory and Applications. Mathematics in Science and Engineering, Academic Press, New York

Fishburn PC, Gehrlein WV (1980) The Paradox of Voting: Effects of individual indifference and intransitivity. J Public Econ 14(1):83-94

García-Lapresta JL, Llamazares B (2000) Aggregation of fuzzy preferences: Some rules of the mean. Soc Choice Welfare 17(4):673-690

García-Lapresta JL, Llamazares B (2001) Majority decisions based on difference of votes. J Math Econ 35(3):463-481

García-Lapresta JL, Llamazares B (2010) Preference intensities and majority decisions based on difference of support between alternatives. Group Decis Negot 19(6):527-542

García-Lapresta JL, Meneses LC (2005) Individual-valued preferences and their aggregation: Consistency analysis in a real case. Fuzzy Sets Syst 151(2):269-284

Gehrlein WV (1983) Condorcet's Paradox. Theor Decis 15(2):161-197

Gehrlein WV (1997) Condorcet Paradox and the Condorcet efficiency of voting rules. Math Jpn 40:173-199

Gehrlein WV, Fishburn PC (1976) Condorcet's Paradox and anonymous preference profiles. Public Choice 26:1-18

Gehrlein WV, Fishburn PC (1980) Robustness of positional scoring over subsets of alternatives. Appl Math Optim 6:241-255

Gehrlein WV, Fishburn PC (1981) Constant scoring rules for choosing one among many alternatives. Qual Quant 15:203-210

Gehrlein WV, Fishburn PC (1983) Scoring rule sensitivity to weight selection. Public Choice 40:249-261

Guilbaud G -TH. (1952) Les théories de l'intérêt général et le problème logique de l'agrégation. Economie Appliquée 5:501-584

Houy N (2007) Some further characterizations for the forgotten voting rules. Math Soc Sci 53(1):111-121

Jones B, Radcliff B, Taber C, Timpone R (1995) Condorcet winners and the Paradox of Voting: Probability calculations for weak preference orders. Am Polit Sci Rev 89(1):133144

Klahr D (1966) A computer simulation of the Paradox of Voting. Am Polit Sci Rev 60(2):384390

Llamazares B (2004) Simple and absolute special majorities generated by OWA operators. Eur J Oper Res 158:707-720

Llamazares B (2006) The forgotten decision rules: Majority rules based on difference of votes. Math Soc Sci 51(3):311-326

Llamazares B (2007) Choosing OWA operator weights in the field of Social Choice. Inf Sci $177: 4745-4756$ 
Llamazares B, García-Lapresta JL (2003) Voting systems generated by quasiarithmetic means and OWA operators, in: J. Fodor, B. De Baets (Eds.), Principles of Fuzzy Preference Modelling and Decision Making, Academia, Press, Ghent, pp. 195-213

Llamazares B, García-Lapresta JL (2008) Extension of some voting systems to the field of gradual preferences, in: H. Bustince, F. Herrera, J. Montero (Eds.), Fuzzy Sets and Their Extensions: Representation, Aggregation and Models, Springer-Verlag, Berlin, pp. $297-315$

Llamazares B, Pérez-Asurmendi P (2015) Triple-acyclicity in majorities based on difference in support. Inf Sci 299:209-220

Llamazares B, Pérez-Asurmendi P, García-Lapresta JL (2013) Collective transitivity in majorities based on difference in support. Fuzzy Sets Syst 216:3-15

McLean I and Urken AB (1995) Classics of Social Choice. Ann Arbor, The University of Michigan Press

Meek BL (1975) A transferable voting system including intensity of preference. Mathématique et Sciences Humaines 50:23-29

Morales JI (1797) Memoria Matemática sobre el Cálculo de la Opinion en las Elecciones. Imprenta Real, Madrid

Nurmi H (1981) Approaches to collective decision making with fuzzy preference relations. Fuzzy Sets Syst 6:249-259

Nurmi H (2008) Fuzzy social choice: a selective retrospect. Soft Computing 12:281-288

Sen AK (1970) Collective Choice and Social Welfare, Holden-Day, San Francisco

Tanino T (1984) Fuzzy preference orderings in group decision making. Fuzzy Sets Syst $12: 117-131$

Tovey CA (1997) Probabilities of preferences and cycles with super majority rules. J Econ Theory 75:271-279

Zadeh LA (1971) Similarity relations and fuzzy orderings. Inform Sci 3(2):177-200 